\title{
Evaluation of sperm DNA damage in bulls by TUNEL assay as a parameter of semen quality
}

\author{
Kumiko TAKEDA ${ }^{1)}$, Kyoko UCHIYAMA ${ }^{2)}$, Masashi KINUKAWA2), Takahiro TAGAMI1), \\ Masahiro KANEDA ${ }^{3)}$ and Shinya WATANABE ${ }^{1)}$ \\ 1) Animal Breeding and Reproduction Research Division, NARO Institute of Livestock and Grassland Science, \\ Ibaraki 305-0901, Japan \\ 2) Maebashi Institute of Animal Science, Livestock Improvement Association of Japan, Inc., Gunma 371-0121, Japan \\ ${ }^{3)}$ Division of Animal Life Science, Tokyo University of Agriculture and Technology, Tokyo 183-8509, Japan
}

\begin{abstract}
Sperm DNA damage affects the conception rate resulting from human assisted reproduction technology. The objective of this study was to adapt the terminal deoxynucleotidyl transferase-mediated dUTP nick end labeling (TUNEL) assay to provide a quality parameter for bull semen based on the detection of sperm DNA damage. Fresh semen was collected from two Japanese Black bulls (A, B) several times over the course of a year, and the percentage of TUNEL-positive spermatozoa (sperm TUNEL index) was determined. Individual differences in semen were detected using the sperm TUNEL index in these bulls $(\mathrm{P}<0.01)$. The sperm TUNEL index of cryopreserved semen obtained from test-mated Japanese Black $(\mathrm{n}=30$, including two bulls with a conception rate lower than $10 \%)$ and Holstein $(\mathrm{n}=34)$ bulls were analyzed. The average sperm TUNEL index and conception rate resulting from artificial insemination (AI) were $4.7 \%$ and $55.7 \%$ for Japanese Black, and $4.9 \%$ and $39.5 \%$ for Holstein, respectively. A weak negative correlation between sperm TUNEL index and conception rate was observed in Holstein bulls $(\mathrm{P}<0.05)$. Semen samples from six bulls with more than $10 \%$ sperm TUNEL index were studied, and these samples showed low sperm viability. However, semen resulting in a very low conception rate did not have a high sperm TUNEL index. Although it would be difficult to predict a low conception rate resulting from AI using the sperm TUNEL index alone, the index can be used as an additional parameter to provide a more comprehensive description of semen quality.
\end{abstract}

Key words: Bull sperm, Conception rate, DNA damage, Semen quality, TUNEL

(J. Reprod. Dev. 61: 185-190, 2015)

$\mathbf{S}^{\mathrm{s}}$ emen quality is routinely assessed according to characteristics of the spermatozoa such as concentration, motility, and morphology that can be observed by microscopy. Even though semen shows normal motility and morphology, some males suffer from extremely low fertility, and a previous report indicated that $15.5 \%$ of idiopathic infertile men had normal semen parameters [1]. It is possible that sperm DNA damage is an underlying factor in successive pregnancy losses and in the failure of assisted conception [2]. DNA damage may involve single-strand breaks or nicks, double-stranded breaks or fragments, deletions, additions, or base modifications. Sperm DNA damage is commonly assayed by the sperm chromatic structure assay (SCSA), single cell gel electrophoresis (Comet assay), and terminal deoxynucleotidyl transferase-mediated dUTP nick end labeling (TUNEL) [3-5]. It remains unclear whether sperm DNA damage is directly related to male fertility [6]; however, assessment of sperm DNA damage is recommended before semen is used for human assisted reproductive technology (ART), including artificial

Received: November 5, 2014

Accepted: January 14, 2015

Published online in J-STAGE: January 27, 2015

(C2015 by the Society for Reproduction and Development

Correspondence: K Takeda (e-mail: kumiko@affrc.go.jp)

This is an open-access article distributed under the terms of the Creative Commons Attribution Non-Commercial No Derivatives (by-nc-nd) License

$<\mathrm{http}: / /$ creativecommons.org/licenses/by-nc-nd/3.0/>. insemination (AI).

The TUNEL assay can detect DNA strand breaks that are mainly induced by reactive oxygen species (ROS) and abortive apoptosis $[7,8]$. Sperm DNA strand breaks occur in each ejaculate and can also be induced by $\operatorname{ROS}[9,10]$. Therefore, the TUNEL assay may be the most appropriate method for assessing sperm DNA strand breaks $[8,11]$. Furthermore, infertile patients whose spermatozoa had a high percentage of DNA damage as detected by TUNEL assay showed significantly lower conception rates [12]. In another study, the percentage of TUNEL-positive spermatozoa (sperm TUNEL index) of semen could differentiate infertile men from fertile men [13]. Sperm TUNEL index was also related to the fertility of fresh semen obtained from Holstein breeding bulls [14], and young Norwegian Red bulls [15].

The objectives of the present study were to verify the efficacy of the sperm TUNEL index for bovine semen quality assessment, and to examine the relationship between the sperm TUNEL index and conception rate resulting from artificial insemination (AI) using cryopreserved semen obtained from Japanese Black and Holstein bulls.

\section{Materials and Methods}

Semen

Fresh semen was obtained from two Japanese Black bulls (A; 14 years-old, and B; 7 years-old) reared in the NARO institute of 
Livestock and Grassland Science (NILGS), 1-3 times per a month from September 2013 to August 2014. Twenty $\mu 1$ of fresh semen was immediately used for the TUNEL assay. The rest of the semen was diluted in extender (composition: $20 \%$ egg-yolk, $0.13 \mathrm{M}$ Tris, $0.05 \mathrm{M}$ citric acid, $0.04 \mathrm{M}$ lactose, $0.04 \mathrm{M}$ raffinose, $0.5 \mathrm{mg} / \mathrm{ml}$ gentamicin, and $600 \mathrm{IU} / \mathrm{ml}$ penicillin $\mathrm{G}$ potassium), and stored at 5 $\mathrm{C}$ for a week [16]. Diluted semen with 7\% glycerol was also frozen and stored in liquid nitrogen until use. Animal experiments were approved by the Committee for the Care and Use of Experimental Animals at NILGS (No.14111041).

Cryopreserved semen from Japanese Black ( $\mathrm{n}=30,14-36$ months old) and Holstein ( $n=34,15-22$ months old) bulls was provided by the Maebashi Institute of Animal Science, Livestock Improvement Association of Japan, Inc. (LIAJ). The semen quality and their conception rate were determined at LIAJ. For the AI service of each bull, the semen collection was performed weekly for one month in most cases. Morphology and motility were determined microscopically as part of the routine semen assessment. Viable spermatozoa and viable spermatozoa with normal acrosomes were assessed with non-lipophilic peanut agglutinin conjugated to FITC (PNA-FITC, Sigma Aldrich, MO, USA) and propidium iodide (PI, Molecular Probes, Life Technologies, OR, USA), and then quantified using flow cytometry (Beckman Coulter, Inc. Brea, CA, USA) [17]. Each one of frozen straws was used for subsequent TUNEL analysis. The AI test was performed once for each Japanese Black bull from 2010 to 2013, and twice for each Holstein bull from 2011 to 2013. Exceptionally, the AI data for one Japanese Black bull (JB2) were accumulated from 1996 to 1997. The conception rates resulting from AI were determined for 32-91 Japanese Black cows/heifers for each Japanese Black bull (a total of 1,234 cows/ heifers) and 124-213 Holstein cows/heifers for each Holstein bull (a total of 5,952 cow/heifers), kept at a private breeding ranch in Japan. The conceptions were examined by a conventional method including 70-day non-return, rectal palpation, and ultrasonography. The conception rate $(\mathrm{CR})$ was calculated by the following equation: $\mathrm{CR}(\%)=\mathrm{nPREG} /(\mathrm{nAI}-\mathrm{nUD}) \times 100$, where $\mathrm{nPREG}$ is the number of pregnancies, $\mathrm{nAI}$ is the number of AI services, and nUD is the number of unidentified pregnancies; these animals were shipped from program prior to pregnancy confirmation. Other reproduction data such as parity of cows/heifers, and estrus synchronization, and number of insemination for each AI service were not obtained.

\section{Sperm preparation}

Frozen-thawed or liquid stored semen was washed twice in Dulbecco's phosphate-buffered saline without calcium chloride and magnesium chloride (PBS-). The wash step was omitted for the fresh semen, and semen was added directly in the following dilution step. Semen was diluted in PBS- to 10 or $20 \times 10^{6}$ spermatozoa $/ \mathrm{ml}$ for all experiments. Sperm smears were prepared on a slide using 15-20 $\mu 1$ of the dilution, slides were air dried and fixed with $2 \%$ $(\mathrm{w} / \mathrm{v})$ paraformaldehyde in PBS- for $30 \mathrm{~min}$.

\section{TUNEL assay}

The amount of DNA fragmentation was determined by TUNEL assay using a commercially available kit (In Situ Cell Death Detection Kit, fluorescein, Roche, Indianapolis, IN, USA), whereby the free
3-OH ends of DNA are labeled with fluorescein conjugated dUTP by the enzyme terminal deoxynucleotidyl transferase. Briefly, the fixed spermatozoa on the slides were washed three times for 5 min with PBS- and permeabilized with $0.1 \%(\mathrm{v} / \mathrm{v})$ Triton X-100 containing $0.1 \%(\mathrm{w} / \mathrm{v})$ sodium citrate for $2 \mathrm{~min}$ on ice. Samples were then incubated in 50- $\mu 1$ of TUNEL reaction mixture for $1 \mathrm{~h}$ at $37 \mathrm{C}$ in a dark and humidified atmosphere. For a positive control, spermatozoa slides were treated with RNase-free DNase I (400 U/ $\mathrm{ml}$, Qiagen, Valencia, CA, USA) at room temperature for $10 \mathrm{~min}$ before incubation with the TUNEL reagent. For a negative control, spermatozoa slides were incubated with the TUNEL reagent in the absence of terminal deoxynucleotidyl transferase. Slides were washed three times with PBS- and cover slips were mounted using mounting medium (VECTASHELD ${ }^{\circledR}$, Vector Laboratories, Burlingame, CA, USA). Positive TUNEL staining was observed under a fluorescence microscope (TE2000U, Nikon, Tokyo, Japan) using the B-2A filter (450-490 nm excitation filter, $505 \mathrm{~nm}$ dichroic mirror, $520 \mathrm{~nm}$ bandpass filter). The sperm TUNEL index was determined by counting the positive and negative stained spermatozoa in each of the ten fields of vision.

\section{Statistical analysis}

Differences between Bull A and B were examined by one-way ANOVA. Statistical analysis shown in Fig. 3 was performed using a paired $t$-test. The relationship between the sperm TUNEL index and conception rate after AI was analyzed by Spearman's rank correlation coefficient.

\section{Results}

\section{An annual variation of sperm TUNEL index}

Fresh semen were collected from two Japanese Black bulls (A, B) over the course of a year (from October 2013 to August 2014), and was examined using the sperm TUNEL index. The results obtained using the sperm TUNEL index changed during the course of the study as shown in Fig. 1a. Individual differences in the sperm TUNEL index were observed between these bulls, and the index of Bull B $(6.9 \pm 3.5 \%)$ was significantly higher than that of Bull $\mathrm{A}(2.3 \pm 1.6 \%)$ [mean \pm standard deviation (SD), $\mathrm{P}<0.01]$. The sperm TUNEL index changed but remained at a low level in Bull A (ranging from 0.3 to $5.6 \%$ ) and was unstable in Bull B (ranging from 2.8 to $15.3 \%$ ) throughout the year. Over the year, the sperm motility of Bull A was better than that of the sperm of Bull B (70+++ vs. $40+++, \mathrm{P}<0.01)$. The sperm motility was altered during the year, especially that from Bull B; however, the results derived from the sperm TUNEL index did not reflect the motility (Fig. 1b).

\section{The sperm TUNEL index under different semen condition}

The sperm TUNEL indices of fresh, frozen-thawed $(n=8)$, and liquid-stored ( $5 \mathrm{C}$ for a week; $\mathrm{n}=6$ ) semen were compared (Fig. 2, and 3). Significant differences in the sperm TUNEL index were detected between "fresh" and "frozen-thawed" semen from Bull A, "fresh" and "liquid-stored" from Bull A, and "fresh" and "frozenthawed" from Bull B $(\mathrm{P}<0.05)$; however, no significant difference in the index was observed between the "fresh" and "liquid-stored" from Bull B $(\mathrm{P}=0.06)$. 
(a)

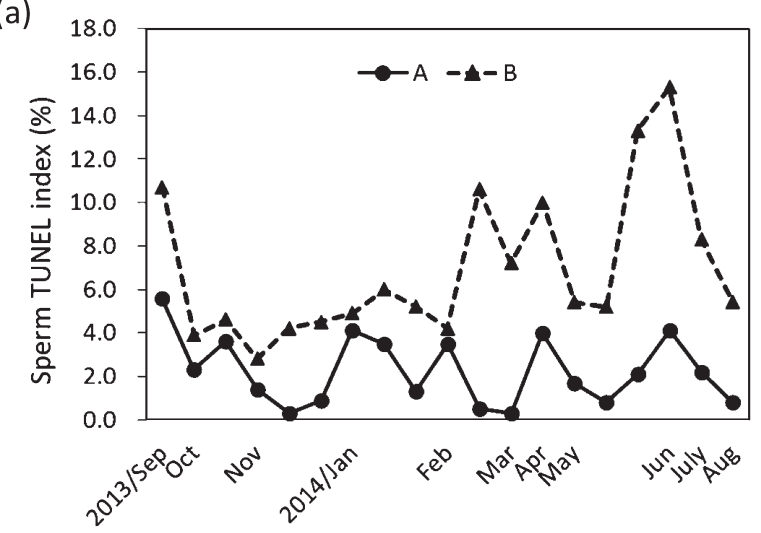

(b)

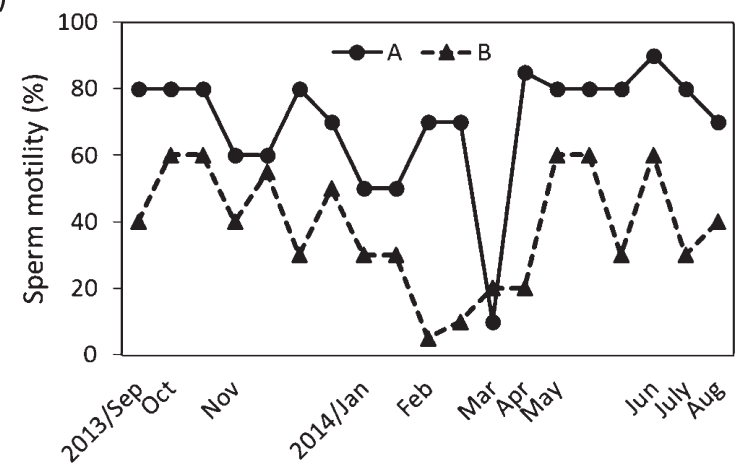

Fig. 1. Sperm TUNEL index (panel a) and sperm motility (panel b) observed in semen from Bull A and B over the course of a year (from September 2013 to August. 2014).

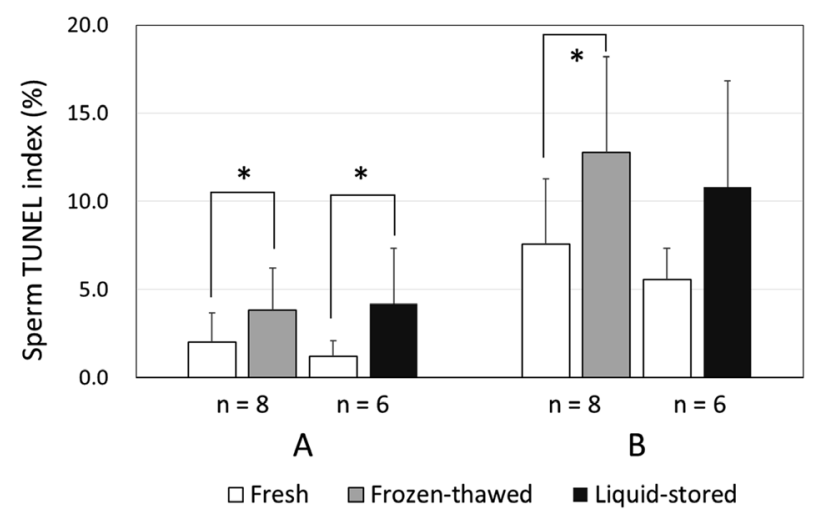

Fig. 3. The mean sperm TUNEL index in semen that was fresh, frozen-thawed, and liquid-stored (5 $\mathrm{C}$ for one week), obtained from Bull A and B. Error bars indicate standard deviation. Asterisks show significant differences between samples $(\mathrm{P}<$ 0.05 ) detected by a paired $t$-test.

The sperm TUNEL index and male fertility

The sperm TUNEL index for frozen-thawed semen obtained from Japanese Black $(\mathrm{n}=30)$ and Holstein $(\mathrm{n}=34)$ bulls was analyzed. The conception rate in these bulls was examined by the AI test. The mean values of the sperm TUNEL index and conception rate were $4.7 \pm 4.0 \%$ and $55.7 \pm 16.2 \%$ for Japanese Black and $4.9 \pm 2.3 \%$ and $39.5 \pm 4.9 \%$ for Holstein bulls, respectively (Table 1). There was no significant correlation between the sperm TUNEL index and conception rate in Japanese Black bulls $(r=-0.27, \mathrm{P}>0.05)$ (Fig. 4a). A weak negative correlation between the sperm TUNEL index and conception rate was observed in Holstein bulls $(\mathrm{r}=-0.33$, $\mathrm{P}<0.05$ ) (Fig. 4b).
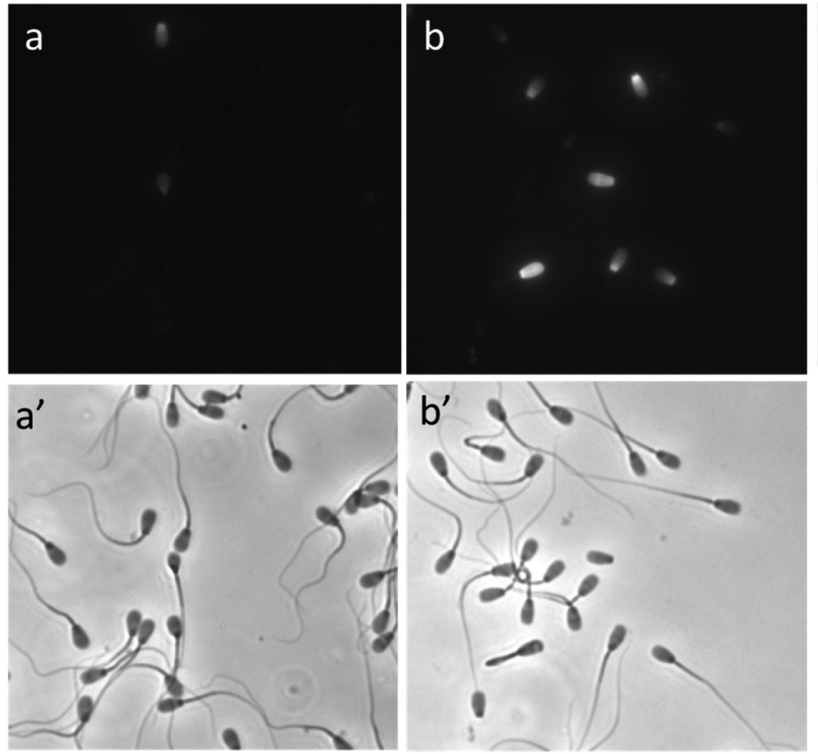
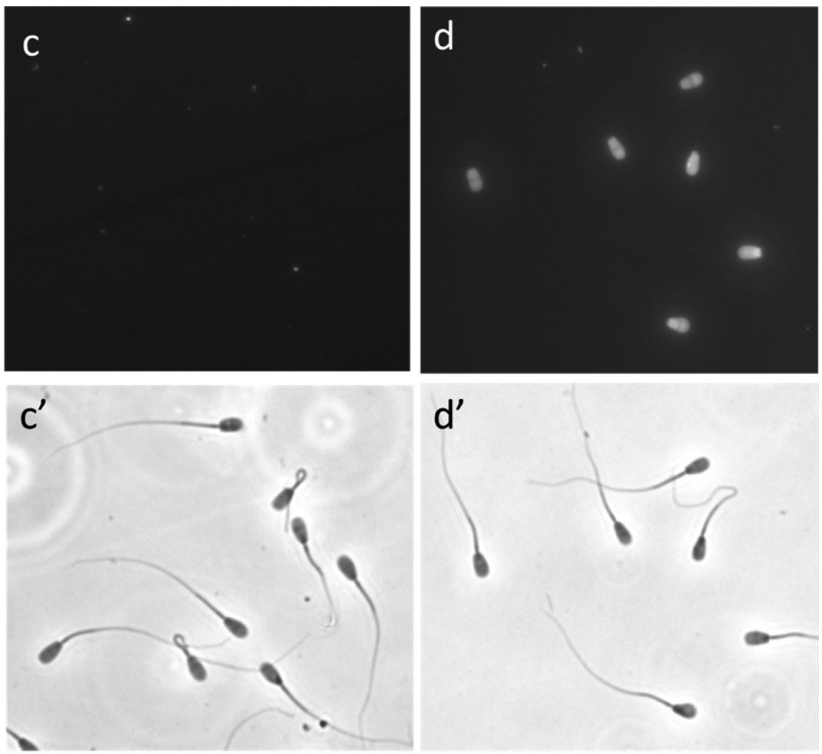
$d^{\prime}$
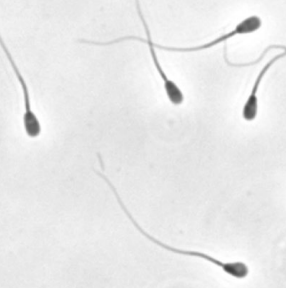

Fig. 2. TUNEL positive spermatozoa obtained from Bull B visualized by fluorescence microscopy. TUNEL positive spermatozoa were detected in fresh semen ( $\left.a, a^{\prime}\right)$, liquid-stored semen ( $5 \mathrm{C}$ for a week; $\left.b, b^{\prime}\right)$, negative control (c, c'), and positive control (d, d'). The upper panels show FITC-stained images, and the lower panels show phase-contrast images. 
Table 1. Intact spermatozoa and those with tail-stump defects observed in semen that resulted in a low conception rate after AI ( $<10 \%)$ or high level of sperm TUNEL index (> 10\%)

\begin{tabular}{|c|c|c|c|c|c|c|c|c|c|}
\hline \multirow{3}{*}{ Bull } & \multicolumn{5}{|c|}{ Spermatozoa analyzed by TUNEL assay } & \multirow{3}{*}{$\begin{array}{c}\text { Viable } \\
\text { spermatozoa } \\
(\%)\end{array}$} & \multirow{3}{*}{$\begin{array}{c}\text { Viable } \\
\text { spermatozoa with } \\
\text { normal acrosomes } \\
(\%)\end{array}$} & \multirow{3}{*}{$\begin{array}{l}\text { Number of } \\
\text { cows/ heifer } \\
\text { used for AI }\end{array}$} & \multirow{3}{*}{$\begin{array}{c}\text { Conception } \\
\text { rate after AI } \\
(\%)\end{array}$} \\
\hline & \multicolumn{2}{|c|}{ All spermatozoa analyzed ${ }^{\text {a) }}$} & \multicolumn{2}{|c|}{$\begin{array}{l}\text { Tail-stump defect } \\
\text { spermatozoa }{ }^{\text {b) }}\end{array}$} & \multirow{2}{*}{$\begin{array}{c}\text { Intact } \\
\text { spermatozoa } \\
\text { Sperm TUNEL } \\
\text { index (\%) }\end{array}$} & & & & \\
\hline & $\begin{array}{c}\text { Number of } \\
\text { spermatozoa analyzed }\end{array}$ & $\begin{array}{l}\text { Sperm TUNEL } \\
\text { index }(\%)\end{array}$ & $\mathrm{b} / \mathrm{a}(\%)$ & $\begin{array}{l}\text { Sperm TUNEL } \\
\text { index }(\%)\end{array}$ & & & & & \\
\hline JB1 & 1234 & 6.8 & 6 & 50.7 & 4.1 & 73 & 65.5 & 33 & 6.1 \\
\hline JB2 & 1270 & 4.8 & 4.6 & 54.7 & 2.3 & 76 & 69.7 & 91 & 8.8 \\
\hline JB3 & 1937 & 15.5 & 5.6 & 67 & 12.4 & 40.5 & 35.5 & 43 & 44.2 \\
\hline JB4 & 1456 & 13.9 & 5.7 & 56.8 & 11.2 & 45 & 29.9 & 42 & 61.9 \\
\hline JB5 & 1523 & 11.8 & 5.8 & 57.5 & 8.8 & 50 & 36.8 & 43 & 60.5 \\
\hline JB6 & 1327 & 10.5 & 4.7 & 66.7 & 7.7 & 43 & 41.8 & 37 & 40.5 \\
\hline JB7 & 1679 & 10.5 & 12.2 & 67.4 & 2.8 & 51.5 & 41.2 & 43 & 53.5 \\
\hline H1 & 886 & 12.2 & 11.3 & 47.6 & 7.7 & 58 & 44.6 & 152 & 32.2 \\
\hline JB-All & & 4.7 & & & & 58 & 50.6 & 41.1 & 55.7 \\
\hline H-All & & 4.9 & & & & 59.6 & 50.4 & 175.1 & 39.5 \\
\hline
\end{tabular}

JB1-2; bulls with low conception rate after AI $(<10 \%)$. JB3 $-7, \mathrm{H1}$; bulls includes high level $(>10 \%)$ of sperm TUNEL index spermatozoa in their semen. JB-All; the mean values of all Japanese Black bulls analyzed $(\mathrm{n}=30)$, H-All; the mean values of all Holstein bulls analyzed $(\mathrm{n}=34)$.

The mean percentages of viable spermatozoa and of viable spermatozoa with normal acrosomes were $58.0 \pm 10.9 \%$ and 50.6 $\pm 13.6 \%$ for Japanese Black and $59.6 \pm 16.2 \%$ and $50.4 \pm 13.4 \%$ for Holstein bulls, respectively (Table 1). A negative correlation between the sperm TUNEL index and the percentage of viable spermatozoa was observed in both Japanese Black $(\mathrm{r}=-0.49, \mathrm{P}<$ $0.01)$ and Holstein bulls $(\mathrm{r}=-0.36, \mathrm{P}<0.05)$. A weak negative correlation between the sperm TUNEL index and the percentage of viable spermatozoa with normal spermatozoa was observed in Japanese Black bulls $(\mathrm{r}=-0.39, \mathrm{P}<0.05)$; however, there was no significant correlation between these parameters in Holstein bulls $(\mathrm{r}=-0.22, \mathrm{P}>0.05)$.

Five Japanese Black bulls (JB3-7) and one Holstein bull (H1) showed more than $10 \%$ sperm TUNEL index (Fig. 4, Table 1). However, bulls with conception rate less than $10 \%$ (JB1; $6.1 \%$, and JB2; $8.8 \%$ ) did not show effectively high levels of sperm TUNEL index (6.8\%, and $4.8 \%$, respectively) (Fig 4., Table 1$)$. The percentage of viable spermatozoa with a normal acrosome was lower in JB3-7 and H1 than in JB1 and JB2 (Table 1). The numbers of spermatozoa with tail-stump defects varied among semen samples; however, in two animals (JB7 and H1) such spermatozoa were detected at levels over $10 \%$ (Table 1 ). The sperm TUNEL index tended to decrease when only intact spermatozoa were counted; especially in JB7 (10.5 vs. 2.8\%). Conversely, the sperm TUNEL index in intact spermatozoa remained high in two animals (JB3 and JB4, 12.4\% and $11.2 \%$, respectively).

\section{Discussion}

The TUNEL assay is one of the most common methods used to assess sperm DNA fragmentation [8, 11]. Several kinds of TUNEL assay kits are available commercially, which are cost effective and standardized by the manufacturers. Although TUNEL-positive spermatozoa can be easily assessed using flow cytometry [8], we analyzed the samples by microscopy in order to assess the relationship between the sperm TUNEL index and tail-stump defects in spermatozoa. Our method can easily identify false positive staining that is not spermatozoon within the sample.

We investigated variation of the sperm TUNEL index in two Japanese Black bulls over the course of a year, and individual differences were observed. To our knowledge, this is the first report that shows changes over one-year in the sperm TUNEL index in bulls. Namely, Bull A scored lower than Bull B in the sperm TUNEL index throughout the year (Fig. 1a). The sperm TUNEL index characteristic is likely to be inherent to each individual semen. The sperm TUNEL index remained at a low level in Bull A semen (ranging from 0.3 to $5.6 \%$ ) and was unstable in Bull B semen (ranging from 2.8 to $15.3 \%$ ) throughout the year. Although the reason is unknown, these results indicated that the sperm TUNEL index can also could vary depending on the collection day for the same bull. Moreover, the results derived from the sperm TUNEL index were not related to semen quality parameters such as sperm motility (Fig. 1b).

The sperm TUNEL index was a successful measure of semen quality using fresh, frozen-thawed, and liquid-stored (at $5 \mathrm{C}$ for one week) semen. The sperm TUNEL index that was observed in two bulls was likely to increase in frozen-thawed or liquid-stored semen (Fig. 3). Our results are in agreement with the findings from human spermatozoa obtained from infertile patients $[18,19]$. In humans, fragmentation of sperm DNA is associated with an increase in oxidative stress during cryopreservation rather than activation of caspases and apoptotic sequelae [19]. This was supported by the present observation that the sperm TUNEL index increased in frozen-thawed, or liquid-stored semen samples. In contrast, some reports have suggested that sperm cryopreservation has no effect on sperm DNA fragmentation in human or bull semen [14, 20, 21]. These incompatibilities between reports might be due to differences in semen freezing conditions such as the composition of semen extender and the freezing procedure used, since sperm damage 
(a)

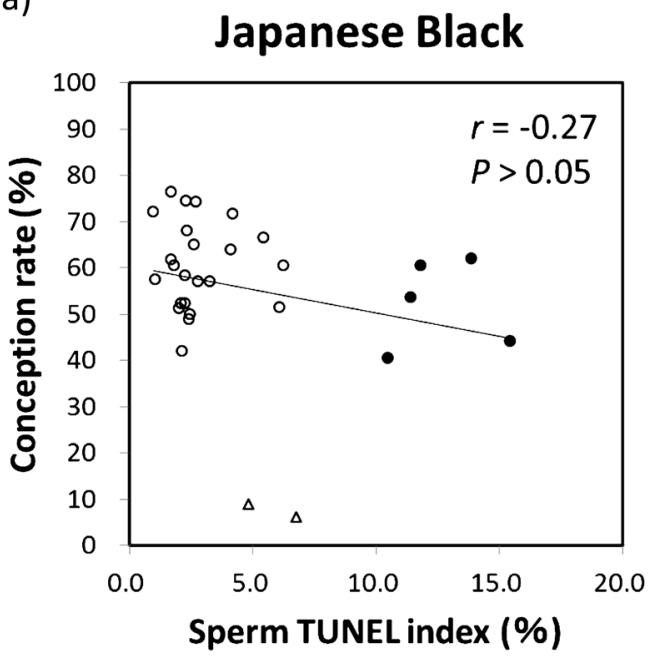

(b)

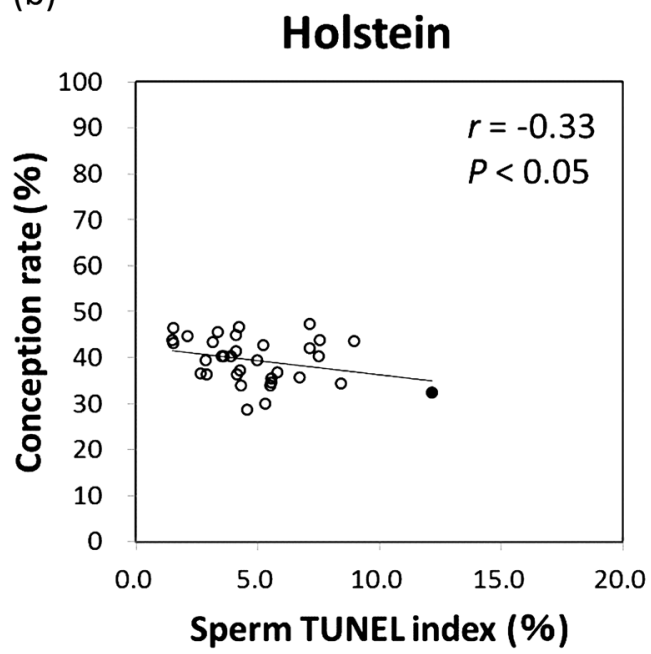

Fig. 4. Relationship between the sperm TUNEL index and the conception rate after artificial insemination of Japanese Black (a) and Holstein bulls (b). Two bulls (JB1-2) with conception rate less than 10\% were indicated as triangles. Six bulls (JB3-7, and H1) with more than 10\% sperm TUNEL index were indicated as black circles. Correlation coefficient $(r)$ and significance $(\mathrm{P}<0.05)$ were analyzed by Spearman's rank correlation coefficient.

occurs during cryopreservation as a result of mechanical stress to the plasma membrane and the generation of excess ROS [22, 23].

In the previous report, Holstein bulls with subfertility had a high sperm TUNEL index ( $25 \%$ and $20 \%$ ), whereas the remaining three bulls that possessed average or high fertility, had less than $15 \%$ [14]. It is thought that sperm with a TUNEL index lower than $20 \%$ may not affect the result of AI. Another research group showed that the TUNEL assay is a valuable tool that can be used to evaluate semen quality in relation to conception rate resulting from AI in young Norwegian red bulls [15]. The bulls that were found to have a sperm TUNEL index less than $4.8 \%$, the odds of AI success were $10 \%$ higher compared with the average. However, using this threshold parameter with our results, the average conception rate did not differ between the Japanese Black bull groups (with the exception of two subfertile bulls, JB1 and JB2) with a lower $(<4.8 \%, \mathrm{n}=20)$ and higher $(>$ $4.8 \%, \mathrm{n}=8)$ sperm TUNEL indices $(60.8 \%$ vs. $54.9 \%, \mathrm{P}>0.05)$. This was also observed between Holstein bulls with lower $(<4.8 \%$, $\mathrm{n}=19)$ and higher $(>4.8 \%, \mathrm{n}=15)$ sperm TUNEL indices $(40.5 \%$ vs. $38.2 \%, \mathrm{P}>0.05)$. This suggests that the threshold parameter of the sperm TUNEL index may be much higher than $4.8 \%$ and needs to be adjusted for Japanese bulls.

Although a weak correlation between the sperm TUNEL index and conception rate was observed in Holstein bulls $(\mathrm{r}=-0.33, \mathrm{P}<$ 0.05 ) (Fig. 4b), semen with a conception rate less than $10 \%$ (JB1 and JB2) did not generate an effective level of sperm TUNEL index. These findings might suggest that the sperm TUNEL index would not be a strict parameter of semen quality in bulls; however, it has been shown that sperm DNA damage induces apoptosis of bovine embryos [24]. The same phenomenon has also been observed in humans[12, 25]. DNA damage in human sperm was not correlated with the fertilization rate, but there was a significantly reduced conception rate in IVF patients inseminated with TUNEL positive spermatozoa [12]. These results also suggest that the TUNEL assay might provide a threshold parameter concerning semen quality. The threshold parameter of the sperm TUNEL index would be more than $20 \%[14]$. In addition, it was suggested that if the semen with this sperm TUNEL index were present at a high level, further inspection would be necessary on other ejaculated semen.

In many studies including this one, the fresh semen was analyzed without washing by centrifugation. Although dead spermatozoa would be included in the assay, such a procedure prevented the exclusion of swim-up spermatozoa with good vitality from the analysis. Actually, a negative correlation between the sperm TUNEL index and the percentages of viable spermatozoa was observed in both Japanese Black and Holstein bulls. The previous report showed that a high incidence of positive TUNEL labeling was observed in spermatozoa with tail stump defects [26]. It is widely accepted that semen containing many abnormal spermatozoa is likely to be associated with low conception rate when used in AI. Table 1 shows that the presence of intact spermatozoa and spermatozoa with tail-stump defect in semen with low conception rate resulted from $\mathrm{AI}(<10 \%)$ and high sperm TUNEL index ( $>10 \%)$. More than half of TUNEL positive spermatozoa exhibited the tail stump defect, which might support the conclusions of the previous report [26]. Although the number of analyses was limited, the percentages of spermatozoa with tail-stump defects did not appear to be a direct factor for their low conception rate resulting from AI. Similarly, the TUNEL index of intact spermatozoa was not directly related to the conception rate. It should be noted that the percentage of viable spermatozoa with normal acrosomes is likely to be negatively correlated with the sperm TUNEL index. Therefore, Percoll density gradient preparations [27] might decrease the sperm TUNEL index of a given semen sample.

In conclusion, the sperm TUNEL index might be effective for assessing the quality of bull semen. It is difficult to predict a low conception rate based on $\mathrm{AI}$; however, the sperm TUNEL index can be used as an additional parameter to provide a more comprehensive 
description of semen quality on the basis of sperm DNA damage. If we can use a sperm quality parameter set to exclude the semen derived from subfertile bulls before performing AI, the cost of cattle production will considerably reduce. It would also help to overcome the worldwide problem of decreasing conception rates resulting from AI, especially in Holstein cows ([28]; the Japanese data is published in http://liaj.or.jp/giken/gijutsubu/seieki/jyutai.htm).

\section{Acknowledgements}

This study was partially supported by funding from the Ministry of Agriculture, Forestry and Fisheries, Japan (Research and development projects for application in promoting new policy of agriculture, forestry and fisheries, 23032).

\section{References}

1. Venkatesh S, Shamsi MB, Deka D, Saxena V, Kumar R, Dada R. Clinical implications of oxidative stress \& sperm DNA damage in normozoospermic infertile men. Indian J Med Res 2011; 134: 396-398. [Medline]

2. Singh A, Agarwal A. The role of sperm chromatin integrity and DNA damage on male infertility. Open Reprod Sci J 2011; 3: 65-71. [CrossRef]

3. Agarwal A, Allamaneni SS. Sperm DNA damage assessment: a test whose time has come. Fertil Steril 2005; 84: 850-853. [Medline] [CrossRef]

4. Ribas-Maynou J, García-Peiró A, Fernández-Encinas A, Abad C, Amengual MJ, Prada E, Navarro J, Benet J. Comprehensive analysis of sperm DNA fragmentation by five different assays: TUNEL assay, SCSA, SCD test and alkaline and neutral Comet assay. Andrology 2013; 1: 715-722. [Medline] [CrossRef]

5. Evenson DP, Wixon R. Clinical aspects of sperm DNA fragmentation detection and male infertility. Theriogenology 2006; 65: 979-991. [Medline] [CrossRef]

6. Zini A, Jamal W, Cowan L, Al-Hathal N. Is sperm DNA damage associated with IVF embryo quality? A systematic review. J Assist Reprod Genet 2011; 28: 391-397. [Medline] [CrossRef]

7. Tremellen K. Oxidative stress and male infertility - a clinical perspective. Hum Reprod Update 2008; 14: 243-258. [Medline] [CrossRef]

8. Sharma R, Masaki J, Agarwal A. Sperm DNA fragmentation analysis using the TUNEL assay. Methods Mol Biol 2013; 927: 121-136. [Medline] [CrossRef]

9. Irvine DS, Twigg JP, Gordon EL, Fulton N, Milne PA, Aitken RJ. DNA integrity in human spermatozoa: relationships with semen quality. J Androl 2000; 21: 33-44. [Medline]

10. Lopes S, Jurisicova A, Sun JG, Casper RF. Reactive oxygen species: potential cause for DNA fragmentation in human spermatozoa. Hum Reprod 1998; 13: 896-900. [Medline] [CrossRef]

11. Payan-Carreira R, Borges P, Mir F, Fontbonne A. Molecular Markers in Sperm Analysis. In: Lemma A (ed.), Success in Artificial Insemination - Quality of Semen and
Diagnostics Employed. Rijeka, Croatia: InTech; 2013: 93-115.

12. Henkel R, Hajimohammad M, Stalf T, Hoogendijk C, Mehnert C, Menkveld R, Gips H, Schill WB, Kruger TF. Influence of deoxyribonucleic acid damage on fertilization and pregnancy. Fertil Steril 2004; 81: 965-972. [Medline] [CrossRef]

13. Sharma RK, Sabanegh E, Mahfouz R, Gupta S, Thiyagarajan A, Agarwal A. TUNEL as a test for sperm DNA damage in the evaluation of male infertility. Urology 2010; 76: 1380-1386. [Medline] [CrossRef]

14. Anzar M, He L, Buhr MM, Kroetsch TG, Pauls KP. Sperm apoptosis in fresh and cryopreserved bull semen detected by flow cytometry and its relationship with fertility. Biol Reprod 2002; 66: 354-360. [Medline] [CrossRef]

15. Waterhouse KE, Haugan T, Kommisrud E, Tverdal A, Flatberg G, Farstad W, Evenson DP, De Angelis PM. Sperm DNA damage is related to field fertility of semen from young Norwegian Red bulls. Reprod Fertil Dev 2006; 18: 781-788. [Medline] [CrossRef]

16. Shiga K, Umeki H, Shimura H, Fujita T, Watanabe S, Nagai T. Growth and fertility of bulls cloned from the somatic cells of an aged and infertile bull. Theriogenology 2005; 64: 334-343. [Medline] [CrossRef]

17. Thomas CA, Garner DL, DeJarnette JM, Marshall CE. Fluorometric assessments of acrosomal integrity and viability in cryopreserved bovine spermatozoa. Biol Reprod 1997; 56: 991-998. [Medline] [CrossRef]

18. Zribi N, Feki Chakroun N, El Euch H, Gargouri J, Bahloul A, Ammar Keskes L. Effects of cryopreservation on human sperm deoxyribonucleic acid integrity. Fertil Steril 2010; 93: 159-166. [Medline] [CrossRef]

19. Thomson LK, Fleming SD, Aitken RJ, De Iuliis GN, Zieschang JA, Clark AM. Cryopreservation-induced human sperm DNA damage is predominantly mediated by oxidative stress rather than apoptosis. Hum Reprod 2009; 24: 2061-2070. [Medline] [CrossRef]

20. Martin G, Sabido O, Durand P, Levy R. Cryopreservation induces an apoptosis-like mechanism in bull sperm. Biol Reprod 2004; 71: 28-37. [Medline] [CrossRef]

21. Duru NK, Morshedi M, Schuffner A, Oehninger S. Cryopreservation-thawing of fractionated human spermatozoa and plasma membrane translocation of phosphatidylserine. Fertil Steril 2001; 75: 263-268. [Medline] [CrossRef]

22. Bailey JL, Bilodeau JF, Cormier N. Semen cryopreservation in domestic animals: a damaging and capacitating phenomenon. J Androl 2000; 21: 1-7. [Medline]

23. Koppers AJ, De Iuliis GN, Finnie JM, McLaughlin EA, Aitken RJ. Significance of mitochondrial reactive oxygen species in the generation of oxidative stress in spermatozoa. $J$ Clin Endocrinol Metab 2008; 93: 3199-3207. [Medline] [CrossRef]

24. Fatehi AN, Bevers MM, Schoevers E, Roelen BA, Colenbrander B, Gadella BM. DNA damage in bovine sperm does not block fertilization and early embryonic development but induces apoptosis after the first cleavages. $J$ Androl 2006; 27: 176-188. [Medline] [CrossRef]

25. Henkel R, Kierspel E, Hajimohammad M, Stalf T, Hoogendijk C, Mehnert C, Menkveld R, Schill WB, Kruger TF. DNA fragmentation of spermatozoa and assisted reproduction technology. Reprod Biomed Online 2003; 7: 477-484. [Medline] [CrossRef]

26. Hendricks KE, Hansen PJ. Can programmed cell death be induced in post-ejaculatory bull and stallion spermatozoa? Theriogenology 2009; 71: 1138-1146. [Medline] [CrossRef]

27. Suzuki K, Geshi M, Yamauchi N, Nagai T. Functional changes and motility characteristics of Japanese Black bull spermatozoa separated by Percoll. Anim Reprod Sci 2003; 77: 157-172. [Medline] [CrossRef]

28. Diskin MG, Morris DG. Embryonic and early foetal losses in cattle and other ruminants Reprod Domest Anim 2008; 43(Suppl 2): 260-267. [Medline] [CrossRef] 